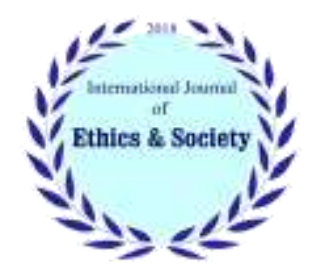

International Journal of Ethics \& Society (IJES)

Journal homepage: www.ijethics.com

Vol. 3, No. 2 (2021)

(Original article)

\title{
Role of Emotional \& Cognitive Empathy, Moral Metacognition \& Quality of Life in Predicting Moral Behavior of Nurses
}

Faezeh Nasirian ${ }^{a}$, Hadi Jafarian ${ }^{b *}$, Yavar Seifi Selseleh ${ }^{c}$

a) Dept. of Psychology, Faculty of Educational Sciences and Psychology, Shabid Beheshti University, Tehran, Iran

b) Dept. of Theology and Islamic Studies, Payame Noor University, Central Tehran, Tehran, Iran

c) Dept. of Psychology, Faculty of Educational Sciences and Psychology, Shiraz. University, Shiraz, Iran

\section{Abstract}

Background: Today, ethics has become an integral part of human life and moral behavior plays an important role in improving the performance and effectiveness of organizations. As a result, this study aimed to determine the role of emotional and cognitive empathy, moral metacognition and quality of life in predicting moral behavior nurses.

Method: The present study was cross-sectional from type of correlation. The study population includes nurses the hospitals of Varamin city in 2019 ( $N=359)$ which from them 200 nurses were selected as a sample by stratified random sampling method. Data were collected by the questionnaires of emotional and cognitive empathy (Reniers et al), moral metacognition (McMahon \& Good), quality of life (WHO) and moral behavior (Swanson \& Hill). Data were analyzed by Pearson correlation coefficient and multiple regression with enter model methods in SPSS-19 software.

Results: The findings showed that emotional empathy, cognitive empathy, moral metacognition and quality of life had a positive and significant relationship with moral behavior of nurses. In addition, the variables of emotional and cognitive empathy, moral metacognition and quality of life were able to predict $56 \%$ of the changes in moral behavior of nurses $(\mathrm{P}<0.01)$.

Conclusion: According to the results, to improve the moral behavior of nurses, their emotional and cognitive empathy, moral metacognition and quality of life can be improved.

Keywords: Emotional and cognitive empathy, Moral metacognition, Quality of life, Moral behavior, Nurses

*Corresponding Author: Email: jafarian1367@yahoo.com

Received: 17 Apr 2021

Accepted: 19 Jun 2021

44

Available at: www.ijethics.com 
Jafarian H. et al.

International Journal of Ethics \& Society (IJES), (2021) Vol. 3, No. 2

\section{Introduction}

Today, ethics has become an integral part of human life and research from many different angles (1). Ethics is a complex structure for which no single definition can be found. For example, the behaviorist approach, unlike the cognitive approach, looks at values and ethics from the perspective of its external influences (2). One of the most popular fields of research on ethics is moral behavior, which means a set of prosocial behaviors with respect for people with different racial and cultural backgrounds, which is learned through punishment and reward (3). Moral behaviors are behaviors that are both for the benefit of others and for the benefit of the individual, and the ultimate criterion for evaluating it is conscience (4). The four factors influencing ethical behaviors include moral sensitivity (interpreting situations and being aware of ethical challenges), moral judgment (ability to recognize and find different solutions to ethical challenges), moral motivation (having a willingness and commitment to ethical behaviors), and moral personality (insistence on doing moral behaviors) (5).

One of the factors related to moral behavior is emotional and cognitive empathy, which is an important concept in the context of social interactions (6). Empathy as a complex and multidimensional concept means the ability to understand others and experience their feelings and cognitions and provide appropriate responses to them, which plays an important role in performing social and moral behaviors (7). Empathy has two parts: emotional and cognitive. Emotional empathy means providing emotional response to the emotional responses of others and creating an emotional reciprocal relationship with them, and cognitive empathy means understanding and recognizing the mental and psychological states and views of others (8). The results showed that empathy had a positive and significant relationship with moral sensitivity and trans-social behaviors (6). In another study, it was found that empathy and moral behavior had a complex and significant relationship (9). Other research results showed a positive and significant relationship between empathy and empathetic concern with moral behavior (10). In another study, it was reported that emotional and cognitive empathy had a significant positive relationship with anonymous, altruistic, emotional, critical, and obsessive societal behaviors (11).

Another factor related to moral behavior is moral metacognition (12). Metacognition is a comprehensive and relatively stable knowledge about beliefs related to the cognitive system that plays an important role in regulating this cognitive system (13). Ethical metacognition reflects an individual's knowledge and awareness of his or her nature, principles, and ethical processes that are used when conducting ethical evaluations and conducting moral behaviors (14). The results showed that ethical metacognition played an important role in moral decision-making and behaviors (12). In another study, it was found that moral metacognition had a significant positive effect on moral behavior (15). Other research results indicate the effective role of moral metacognition in predicting moral behavior (16).

Another factor related to moral behavior is quality of life (17), which as an important part of feeling healthy includes physical, psychological and social aspects and includes physical, financial, emotional, psychological and social satisfaction (18). Quality of life is a comprehensive and multidimensional concept that means the perception of cultural contexts based on personal goals, expectations and standards and plays an important role in psychological health (19). The results showed that there was a positive and significant relationship between quality of work life and moral behavior (17). In another study, it was found that the quality of life of employees had a positive and significant relationship with ethical beliefs and ethical lifestyle (20). Other research results showed a direct and positive effect of quality of work life on ethical culture (21).

It is very important to study the ethical behavior of employees because of its role and importance in improving the performance and effectiveness of organizations and its importance in health care organizations, especially nurses because of their role in caring for patients and maintaining and promoting their health. In addition, although much research 
has been done on moral behavior, it has paid less attention to the role of emotional and cognitive empathy, moral metacognition, and quality of life. Therefore, one of the existing research gaps is not paying attention to the role of these variables and their ability to predict ethical behavior. Another important point is which of these variables plays a greater role in this prediction. As a result, this study aimed to determine the role of emotional and cognitive empathy, moral metacognition and quality of life in predicting moral behavior of nurses.

\section{Material \& Methods}

The present study was a cross-sectional-correlation study. Population of nurses in Varamin hospitals included Shahid Mofteh and 15 Khordad hospitals in 2019 ( $\mathrm{N}=359)$. The sample size was calculated based on Cochran's formula ( $\mathrm{n}=186)$, but finally 200 nurses were selected as sample group, which were selected by stratified random sampling. In this method, the list of nurses was sampled by gender and sampled in the same proportion. Inclusion criteria include willingness to participate in research, minimum associate education, not receiving psychological services in the last three months, no addiction and use of psychiatric drugs and the occurrence of stressful events such as divorce and death of loved ones in the last three months and exclusion criteria include refusal to complete the questionnaires and failure to answer more than ten percent of the items. The results of the number and frequency of nurses' demographic information were presented in table 1.

In this study, in addition to the demographic information form, the following questionnaires were used to collect data.

Emotional and cognitive empathy questionnaire: This questionnaire was designed with 31 items in two dimensions of emotional empathy (12 items) and cognitive empathy (19 items). The items are obtained using a four-point Likert scale from one to four scores, and the score of each dimension is obtained with the total score of its constituent items, so the range of emotional empathy scores between 12 to 48 and cognitive empathy scores between 19 and 76 and higher scores indicate more that feature. The construct validity of the instrument was confirmed by factor analysis and its reliability was obtained by Cronbach's alpha for emotional empathy 0.72 and cognitive empathy 0.87 (22). In Iran, the reliability of the instrument with Cronbach's alpha method for emotional empathy was 0.73 and cognitive empathy was 0.79 (23). In the present study, the reliability value was calculated by Cronbach's alpha method for emotional empathy 0.75 and cognitive empathy 0.84 .

\section{Table1: Number and percentage of frequency} of demographic information in nurses

\begin{tabular}{|l|l|l|l|}
\hline \multirow{3}{*}{ Variables } & Description & No & Percentage \\
\hline \multirow{3}{*}{ Marriage } & Male & 111 & 55.5 \\
\cline { 2 - 4 } & female & 89 & 44.5 \\
\hline \multirow{3}{*}{ Education } & Married & 173 & 86.5 \\
\cline { 2 - 4 } & single & 27 & 13.5 \\
\hline \multirow{5}{*}{ Work experience } & A.A.S & 24 & 12 \\
\hline \multirow{5}{*}{} & B.A & 163 & 81.5 \\
\cline { 2 - 4 } & M.A & 13 & 6.5 \\
\hline & $1-5 y s$ & 28 & 14 \\
\cline { 2 - 4 } & 6-10ys & 43 & 21.5 \\
\cline { 2 - 4 } & $11-15 y s$ & 23 & 11.5 \\
\cline { 2 - 4 } & $16-20 y s$ & 47 & 23.5 \\
\cline { 2 - 4 } & $21-25 y s$ & 38 & 19 \\
\cline { 2 - 4 } & $26-30 y s$ & 21 & 10.5 \\
\hline
\end{tabular}

Moral metacognition questionnaire: This questionnaire was designed with 20 items. The items are obtained using a five-point Likert scale from one to five scores, and the instrument score is obtained with the total score of the items, so the range of scores between 20 and 100 and a higher score indicates greater moral metacognition. The validity of the instrument structure was confirmed by factor analysis and its reliability was obtained by Cronbach's alpha method of 0.94 (13). In Iran, the reliability of the instrument was reported to be 0.90 by Cronbach's alpha method (15). In the present study, the reliability value was calculated by Cronbach's alpha method of 0.87 .

Quality of Life Questionnaire: This questionnaire was designed with 26 items. The items are obtained using a five-point Likert scale from one to five scores and the instrument score with the total score of the items, so the range of scores between 26 to 130 and higher scores indicate a more desirable quality of life. The content validity of the instrument was confirmed by experts and the construct 
validity was confirmed by factor analysis and its reliability was obtained by Cronbach's alpha method in different countries above 0.70 (24). In Iran, the reliability of the instrument was reported to be 0.79 by Cronbach's alpha method (25). In the present study, the reliability value was calculated by Cronbach's alpha method of 0.83 .

Moral Behavior Questionnaire: This questionnaire was designed with 15 items. Items are obtained using a five-point Likert scale from one to five scores, and the instrument score is obtained with the total score of the items, so a range of scores between 15 and 75 and a higher score indicates more desirable moral behavior. The content validity of the instrument was confirmed by experts and its reliability was obtained by Cronbach's alpha method (0.96) (26). In Iran, the reliability of the instrument with Cronbach's alpha method was reported to be 0.76 (27). In the present study, the reliability value was calculated by Cronbach's alpha method (0.79).

The research was conducted in such a way that after coordination with the managers and officials of Shahid Mofteh and 15 Khordad Varamin hospitals, a list of nurses by gender was prepared and sampling was performed. For example, the importance and necessity of research was stated and they were reassured about the observance of ethical points such as confidentiality, privacy of personal information, analysis of data in general, and so on. Samples were then asked to carefully study the questionnaire items and answer them all. It should be noted that they were told that there is no right or wrong answer and the best answer is the one that reflects their true situation.

Data were collected by the above tools and analyzed by Pearson correlation coefficient and multiple regression with simultaneous model in SPSS-19 software at a significance level of 0.05 .

\section{Results}

There was no shedding in the samples and analyzes were performed for 200 nurses with a mean and standard deviation of $41.19 \pm 5.27$ years. Mean, standard deviation and correlation coefficients of emotional and cognitive empathy, moral metacognition, quality of life and moral behavior of nurses were presented in table 2 .

According to the results of table 2, emotional empathy $(\mathrm{r}=0.37)$, cognitive empathy $(\mathrm{r}=0.41)$, moral metacognition $(r=0.52)$ and quality of life $(r$ $=0.29)$ have a positive relationship with nurses' moral behavior and were significant $(\mathrm{P}<0.01)$.

Table 2: Mean, standard deviation and correlation coefficients of research variables in nurses

\begin{tabular}{|c|c|c|c|c|c|c|c|}
\hline Variable & Mean & SD & $\mathbf{1}$ & $\mathbf{2}$ & $\mathbf{3}$ & $\mathbf{4}$ & $\mathbf{5}$ \\
\hline Emotional empathy & 33.29 & 4.28 & 1 & & & & \\
\hline Cognitive empathy & 44.75 & 5.92 & 0.25 & 1 & & & \\
\hline Moral metacognition & 56.13 & 7.25 & 0.44 & 0.38 & 1 & & \\
\hline Quality of life & 87.80 & 9.64 & 0.30 & 0.36 & 0.27 & 1 & \\
\hline Moral behavior & 52.21 & 6.90 & 0.37 & 0.41 & 0.52 & 0.29 & 1 \\
\hline
\end{tabular}

Examination of regression hypotheses showed that the hypothesis of normality based on Kolmogorov-Smirnov test values was confirmed due to significance greater than 0.05 for all five variables of emotional empathy, cognitive empathy, moral metacognition, quality of life and moral behavior. The multiple linearity hypothesis based on variance inflation factor for the predictor variables was rejected by being in the range of 1.24 to 1.83 due to being smaller than 10 . The correlation hypothesis of residuals in the range of 1.5 to 2.5 was confirmed. As a result, there were conditions for regression. A summary of the results of multiple regression with a simultaneous model for predicting nurses' moral behavior based on the variables of emotional and cognitive empathy, moral metacognition and quality of life was presented in table 3.

According to the results of table 3 , the variables of emotional and cognitive empathy, moral metacognition and quality of life could significantly predict $56 \%$ of the changes in nurses' moral behavior, which according to beta content, the share of 
moral metacognition was higher than other varia-

bles $(\mathrm{P}<0.001)$.

Table 3: Summary of multiple regression results with simultaneous model for predicting ethical behavior in nurses

\begin{tabular}{|c|c|c|c|c|c|c|c|c|}
\hline Variable & $\mathbf{R}$ & $\mathbf{R}^{2}$ & df & F & Sig. & Beta & t value & Sig. \\
\hline Emotional empathy & & & & & & 0.25 & 3.41 & 0.001 \\
\hline Cognitive empathy & & & 4 & & & 0.28 & 3.77 & 0.001 \\
\hline Moral metacognition & 0.75 & 0.56 & 195 & 23.48 & 0.001 & 0.36 & 4.53 & 0.001 \\
\hline Quality of life & & & & & & 0.21 & 3.08 & 0.001 \\
\hline
\end{tabular}

\section{Discussion}

Considering the role and importance of moral behavior of nurses in organizational effectiveness, patients' satisfaction with the hospital and their success and job performance, this study was conducted to determine the role of emotional and cognitive empathy, moral metacognition and quality of life in predicting moral behavior of nurses.

Findings showed that emotional and cognitive empathy had a positive and significant relationship with moral behavior of nurses, which was consistent with the results of previous studies $(6,9$ 11). In explaining these results, it can be said that empathy plays a motivating role in moral behaviors in interpersonal situations. Because cognitive and emotional empathy refers to understanding and predicting what others may think, feel, or do, and how to respond to them. As a result, empathy makes people sensitive to the needs of others and the situational factors that may create those needs, and it would no doubt be ethical to engage in such behaviors. Thus, empathy (cognitive and emotional) increases moral behavior (9). Another explanation is that emotional and cognitive empathy is an essential element for successful interpersonal actions, emotional response to the feelings of others and the underlying process of psychological change that reduces work problems by sharing others 'emotional states, receiving regular feedback from interpersonal relationships and awareness of others' feelings. Improves social relationships and appropriate and moral relationships with others. Empathy is also an important ability that coordinates nurses with patients' feelings and thoughts, connects them to the social world, and prevents them from deviating from their job duties. Since one of the prominent characteristics of nurses is the ability to empathize with patients and perform appropriate and ethical behaviors with them, so with increasing emotional and cognitive empathy, the amount of moral behavior in nurses increases (23).

Other findings showed that ethical metacognition had a positive and significant relationship with nurses' moral behavior, which was consistent with the results of previous studies $(12,15-16)$. In explaining these results, it can be said that people's awareness of their moral cognitions is an important prerequisite for using moral behaviors, and people with such characteristics organize their activities by examining, designing, guiding, reviewing, and evaluating cognitive activities and thinking about them. Regulating cognitive processes and activities increases the success of behaviors and reduces failure in them, so with increasing moral metacognition, the amount of moral behavior improves (12). Another explanation is that based on moral agency, people who in each moral situation anticipate their own and others' reactions and think about their actions and consequences, consider different methods of action, and measure the value of each method are more likely to engage in behaviors. They do morally. In other words, these people perform actions that create satisfaction and value for them and avoid actions that cause them dissatisfaction, humiliation, blame and harm to others. Thus, having such characteristics, which are indicators of moral metacognition, increases the performance of moral behaviors (15). Other findings showed that quality of life had a positive and significant relationship with nurses' 
moral behavior, which was consistent with the results of previous studies (17, 20-20). Explaining these results, it can be said that the quality of work life increases the vitality, hope, happiness and job performance of employees and provides the basis for ethical behaviors. Quality of life is achieved through fair payments, appropriate and flexible working hours, cooperation and consultation with employees in organizational decisions, using the real capabilities of employees and having expectations based on it, and increases life satisfaction and ethical behaviors (17). Another explanation is that the quality of work life is a valuable strategy to improve the ethical culture, and if organizations can increase the quality of life and job satisfaction of employees and provide conditions in the workplace so that they feel justice, equality, security, growth and promotion opportunities for Expressing their abilities and creativity, we can see an increase in employee performance and their growth and dynamism (21). As a result, moral behavior increases with the quality of life.

In addition, the findings showed that emotional and cognitive empathy, moral metacognition and quality of life had a significant role in predicting nurses' moral behavior. In explaining these results, it can be said that people have the ability to provide emotional responses to the emotional responses of others and establish an emotional reciprocal relationship with them (emotional empathy), understanding mental and psychological states and views of others (cognitive empathy), relatively comprehensive knowledge and awareness and the stable nature of the person, their moral principles and processes (moral metacognition) and the desired perception of cultural contexts based on personal goals, expectations and standards (quality of life) in personal and professional life face less stressful events, usually alone or with helping others overcome challenges by using adaptive coping strategies. They usually have higher mental health and higher life and job satisfaction, all of which can play an effective role in improving adaptive behaviors, including moral ones, in nurses. Another explanation is that emotional and cognitive empathy, moral metacognition and quality of life are positively correlated with many positive psychological indicators such as happiness, hope, life satisfaction, health, etc. that have the ability to predict moral behavior. As a result, when these variables such as emotional and cognitive empathy, moral metacognition and quality of life enter the regression equation, they can significantly predict the moral behavior of nurses.

\section{Conclusion}

The results showed a positive and significant relationship between emotional empathy, cognitive empathy, moral metacognition and quality of life with moral behavior of nurses and the effective role of these variables in significantly predicting their moral behavior. According to these results, planning to improve the ethical behavior of nurses by improving emotional and cognitive empathy, ethical metacognition and quality of life by hospital specialists and planners is essential. Therefore, health professionals can improve the level of ethical behavior of nurses by increasing the emotional and cognitive empathy, moral metacognition and quality of life of nurses with the help of workshops and improving working conditions.

\section{Ethical Consideration}

In the present study, ethical principles and considerations such as confidentiality of personal information, data analysis in general, participants' awareness of the importance and necessity of research, anonymity of questionnaires, signing an informed consent form to participate in research and having the freedom to refuse to complete questionnaires was respected.

\section{Acknowledgement}

The authors consider it necessary to thank the officials of Shahid Mofteh and 15 Khordad Varamin hospitals for their cooperation with the researchers and the nurses participating in the study for completing the questionnaires accurately. 


\section{References}

1. Olson JG, McFerran B, Morales AC, Dahl DW (2021). How income shapes moral judgments of prosocial behavior. International Journal of Reseanh in Marketing, 38(1): 120135.

2. Nami P, Pasha Sharifi H, Mirhashemi M (2020). Comparison of the ethical status of public and non-profit primary school students. International Journal of Ethics \& Society, 2(3): 1-9.

3. Toure-Tillery M, Light AE (2018). No self to spare: How the cognitive structure of self-influences moral behavior. Organizational Behavior and Human Decision Processes, 147: 4864.

4. Andersch H, Arnold C, Seemann A, Lindenmeier J (2019). Understanding ethical purchasing behavior: Validation of an enhanced stage model of ethical behavior. Journal of Retailing and Consumer Services, 48: 50-59.

5. Purzycki BG, Pisor AC, Apicella C, Atkinson Q, Cohen E, Henrich J, et al. (2018). The cognitive and cultural foundations of moral behavior. Evolution and Human Behavior, 39(5): 490-501.

6. Suazo I, Perez-Fuentes MD, Jurado MDM, Martinez AM, Marquez MDS, Martin ABB, et al. (2020). Moral sensitivity, empathy and prosaically behavior: Implications for humanization of nursing care. International Journal of Emvironmental Research and Public Health, 17(8914): 1-14.

7. Berger P, Bitsch F, Jakobi B, Nagels A, Straube B, FAlkenberg I (2019). Cognitive and emotional empathy in patients with schizophrenia spectrum disorders: A replication and extension study. Psychiatry Research, 276: 56-59.

8. Abramson L, Uzefovsky F, Toccaceli V, Knafo-Noam A (2020). The genetic and environmental origins of emotional and cognitive empathy: Review and meta-analyses of twin studies. Neuroscience \& Biobehavioral Reviens, 114: 113-133.

9. Decety J, Cowell JM (2014). Friends or foes: Is empathy necessary for moral behavior? Perspectives on Psychological Science, 9(5): 525-537.

10. Rezayi Z, Hejazi E, Kareshki H (2020). The moderating role of empathy in the relationship between moral judgment and moral behavior. Journal of Psychology, 24(2): $167-$ 183. (In Persian)

11. Torkman Malayeri M, Sheikholeslami R (2019). Empathy and pro-social behavior: The mediating role of moral emotions. Journal of Developmental Psychology: Iranian Psychologists, 15(59): 261-272. (In Persian)

12. Bajovic M, Rizzo K (2021). Meta-moral cognition: bridging the gap among adolescents' moral thinking, moral emotions and moral actions. International Journal of Adolescence and Youth, 26(1): 1-11.

50

Available at: www.ijethics.com
13. McMahon JM, Good DJ (2016). The moral metacognition scale: Development and validation. Ethics \& Behavior, 26(5): 357-394.

14. Schmidt MFH, Gonzalez-Cabrera I, Tomasello M (2017). Children's developing metaethical judgments. Journal of Experimental Child Psychology, 164: 163-177.

15. Hassannia S, Sheikholeslami R, Yousefi F, Khormaei F (2020). Mediating role of ethical metacognition in relationship between personality and ethical behaviors. The Journal of Ethics in Science and Technology, 14(4): 47-55. (In Persian)

16. Nikdel F, Dehghan M, Noushadi N (2018). The mediating role of moral reasoning on the relationship between self-concept and moral metacognition with moral behavior. Educational Psychology, 13(46): 123-143. (In Persian)

17. Pio RJ, Lengkong FDJ (2020). The relationship between spiritual leadership to quality of work life and ethical behavior and its implication to increasing the organizational citizenship behavior. Journal of Management Development, 39(3): 293-305.

18. Slocum RB, Villano JL. (2021). Narrative medicine applications for neuro-oncology patient identity and quality of life. Journal of Clinical Neuroscience, 83: 8-12.

19. Puolakka K, Pitkanen A (2019). Effectiveness of psychosocial interventions on quality of life of patients with schizophrenia and related disorders: A systematic review. $A r$ chives of Psychiatric Nursing, 33(5): 73-82.

20. Moradi M, Karani H (2020). Analyzing the relationship between ethical beliefs and employees' quality of life with the mediating role of ethical lifestyle. Supervision \& Inspection, 14(53): 101-128. (In Persian)

21. Karimi A (2019). Analysis the relationship between quality of work life and job satisfaction with ethical culture. The Journal of Ethics in Science and Technology, 14(3): 91-98. (In Persian)

22. Reniers RL, Corcoran R, Drake R, Shryane NM, Vollm BA (2011). The QCAE: A questionnaire of cognitive and affective empathy. Journal of Personality Assessment, 93(1): 84 95.

23. Kharidar Atigh S, Zeinali A (2019). The role of emotional and cognitive empathy in predicting job and burnout satisfaction of nurses. Journal of Nursing Education, 7(5): 45-51. (In Persian)

24. World Health Organization (2010). The world health organization quality of life (WHOQOL)-BREF. World Health Organization, Geneva.

25. Rajaei Z, Shafizadeh H, Babaeiamiri N, Amirfakhraei A, Ashoori J (2017). The role of job burnout, perceived social support and psychological hardiness in predicting quality of life of nurses. Iran Journal of Nursing, 30(106): 1-10. (In Persian) 
26. Swanson HL, Hill G (1993). Metacognitive aspects of moral reasoning and behavior. Adolescence, 28(111): 711735 .
27. Ashoori J (2016). The relationship of nurse's moral reasoning, moral behavior and health with their moral metacognition. Journal of Medical Ethics, 9(34): 55-76. (In Persian). 